\title{
DOMINATED SPLITTING WITH STABLY EXPANSIVE
}

\author{
MAnseob LeE
}

Abstract. In this paper, we show that if a transitive set $\Lambda$ is $C^{1}$-stably expansive, then $\Lambda$ admits a dominated splitting.

\section{INTRODUCTION}

In this paper, we study dominated splitting - a weak form of hyperbolicity. More precisely, using results of [2] and [3], we show that if a closed set have the some property then it admits dominated splitting.

Let $M$ be a closed $C^{\infty}$ manifold, and let $\operatorname{Diff}(M)$ be the space of diffeomorphisms of $M$ endowed with the $C^{1}$-topology. Denote by $d$ the distance on $M$ induced from a Riemannian metric $\|\cdot\|$ on the tangent bundle $T M$. Let $f \in \operatorname{Diff}(M)$, and let $\Lambda \subset M$ be a closed $f$-invariant set. We say that $\left.f\right|_{\Lambda}$ is expansive if there is a constant $e>0$ such that for any pair of distinct points $x, y \in \Lambda, d\left(f^{n}(x), f^{n}(y)\right)>e$ for some $n \in \mathbb{Z}$. Let $f \in \operatorname{Diff}(M)$, and let $\Lambda \subset M$ ba a closed $f$-invariant set. We say that $\Lambda$ is locally maximal if there is a compact neighborhood $U$ of $\Lambda$ such that

$$
\bigcap_{n \in \mathbb{Z}} f^{n}(U)=\Lambda(U)
$$

We say that $\Lambda$ admits a dominated splitting if the tangent bundle $T_{\Lambda} M$ has a continuous $D f$-invariant splitting $E \oplus F$ and there exists constant $C>0$ and $0<\lambda<1$ such that

$$
\left\|\left.D_{x} f^{n}\right|_{E(x)}\right\| \cdot\left\|\left.D_{x} f^{-n}\right|_{F\left(f^{n}(x)\right)}\right\| \leq C \lambda^{n}
$$

for all $x \in \Lambda$ and $n \geq 0$.

Received by the editors June 1, 2010. Revised October 16, 2010. Accepted September 2, 2011. 2000 Mathematics Subject Classification. Primary 37D30, Secondary 37 B35.

Key words and phrases. expansive, transitive set, dominated splitting, chain transitive set.

This work was supported by Basic Science Research Program through the National Research Foundation of Korea(NRF) funded by the Ministry of Education, Science and Technology (No. 2011-0007649). 
The following definition is in [5].

Definition 1.1. We say that an $f$-invariant set $\Lambda$ is $C^{1}$-stably expansive if there exists a $C^{1}$-neighborhood $\mathcal{U}(f)$ of $f$ and a compact neighborhood $U$ of $\Lambda$ such that:

- $\Lambda(U)=\bigcap_{n \in \mathbb{Z}} f^{n}(U)$,

- for any $g \in \mathcal{U}(f),\left.g\right|_{\Lambda_{g}(U)}$ is expansive, where $\Lambda_{g}(U)=\bigcap_{n \in \mathbb{Z}} g^{n}(U)$ is called the continuation of $\Lambda$.

Let $f \in \operatorname{Diff}(M)$, and let $\Lambda \subset M$ be a closed $f$-invariant set. Then we say that $\Lambda$ is called a transitive set if there exists a point $x \in \Lambda$ such that $\omega(x)=\Lambda$. Mañè [6] studied the case in which for $f \in \operatorname{Diff}(M)$ there is a $C^{1}$-neighborhood $\mathcal{U}(f)$ of $f$ such that for any $g \in \mathcal{U}(f), g$ is expansive. He proved in the case $f$ is quasi-Anosov, that is, for all $v \in T M, v \neq 0$, the set $\left\{D f^{n}(v) \|: n \in \mathbb{Z}\right\}$ is bounded. Thus we can restate the above facts are follows.

Theorem A. $M$ is $C^{1}$-stably expansive if and only if $f$ satisfies quasi-Anosov.

In this paper, we get a problem which if a transitive set $\Lambda$ is $C^{1}$-stably expansive then is $\Lambda$ is hyperbolic? Unfortunately, it is not true. Indeed, for any hyperbolic periodic points $p, q \in \Lambda$, we don't know that $W^{s}(p) \pitchfork W^{u}(q) \neq \phi$ and $W^{u}(p) \pitchfork$ $W^{s}(q) \neq \phi$. Therefore, our aim is to characterize closed sets by making use of the $C^{1}$-stably expansive property. We are now in position to state main theorem.

Theorem B. Let $\Lambda$ be a transitive set. If $\Lambda$ is $C^{1}$-stably expansive, then $\Lambda$ admits a dominated splitting.

\section{Introduction Some Results.}

We use Mãné's result which is on a uniformly family of periodic sequences of linear maps of $\mathbb{R}^{n}(n=\operatorname{dim} M)$. Let $G L(n)$ be the group of linear isomorphisms of $\mathbb{R}^{n}$. If a sequence $\xi: \mathbb{Z} \rightarrow G L(n)$ is periodic if there is $k>0$ such that $\xi_{j+k}=\xi_{j}$ for $k \in \mathbb{Z}$. We call a finite subset $\mathcal{A}=\left\{\xi_{i}: 0 \leq i \leq k-1\right\} \subset G L(n)$ is a periodic family with period $k$. For a periodic family $\mathcal{A}=\left\{\xi_{i}: 0 \leq i \leq n-1\right\}$, we denote

$$
\mathcal{C}_{\mathcal{A}}=\xi_{n-1} \circ \xi_{n-2} \circ \cdots \circ \xi_{0} .
$$

Definition 2.1. We say that the periodic family $\mathcal{A}=\left\{\xi_{i}: 0 \leq i \leq n-1\right\}$ admits a l-dominated splitting, if there is a splitting $\mathbb{R}^{n}=E \oplus F$ which satisfies:

(a) $E$ and $F$ are $\mathcal{C}_{\mathcal{A}}$ invariant, i.e., $\mathcal{C}_{\mathcal{A}}(E)=E$ and $\mathcal{C}_{\mathcal{A}}(F)=F$,

(b) For any $k=0,1,2, \ldots$, 


$$
\frac{\left\|\left.\xi_{k+l-1} \circ \cdots \circ \xi_{k+1} \circ \xi_{k}\right|_{E_{k}}\right\|}{m\left(\left.\xi_{k+l-1} \circ \cdots \circ \xi_{k+1} \circ \xi_{k}\right|_{F_{k}}\right)} \leq \frac{1}{2}
$$

where

$$
E_{k}=\xi_{k-1} \circ \xi_{k-2} \circ \cdots \circ \xi_{0}(E)
$$

and

$$
F_{k}=\xi_{k-1} \circ \xi_{k-2} \circ \cdots \circ \xi_{0}(F) .
$$

We know that the following theorems for periodic family from [3].

Theorem 2.2. Given any $\epsilon>0$ and $K>0$, there is $n_{1} \geq 0$ which satisfies the following property: given any periodic family $\mathcal{A}=\left\{\xi_{i}: 0 \leq i \leq n-1\right\}$ which satisfies the period $n \geq n_{1}$ and $\max \left\{\left\|\xi_{i}\right\|,\left\|\xi_{i}^{-1}\right\|\right\} \leq K$, for all $i=0,1, \cdots, n-1$, one can find a periodic family $\mathcal{B}=\left\{\zeta_{i}: 0 \leq n-1\right\}$ such that $\max \left\{\left\|\zeta_{i}-\xi_{i}\right\|,\left\|\zeta_{i}^{-1}-\xi_{i}^{-1}\right\|\right\}<\epsilon$, for any $i=0,1, \cdots, n-1$, and $\operatorname{det}\left(\mathcal{C}_{\mathcal{A}}\right)=\operatorname{det}\left(\mathcal{C}_{\mathcal{B}}\right)$ and the eigenvalues of $\mathcal{C}_{\mathcal{B}}$ are all real, multiplicity one and different moduli.

Theorem 2.3. Given any $\epsilon>0$ and $K>0$, there is positive integers $n_{2} \geq 0$ and $l \geq 0$ which satisfies the following property: given any periodic family $\mathcal{A}=\left\{\xi_{i}\right.$ : $0 \leq i \leq n-1\}$ which satisfies the period $n \geq n_{2}$ and $\max \left\{\left\|\xi_{i}\right\|,\left\|\xi_{i}^{-1}\right\|\right\} \leq K$, for all $i=0,1, \cdots, n-1$, if $\mathcal{A}$ does not admits any l-dominated splitting, then one can find a periodic family $\mathcal{B}=\left\{\zeta_{0}, \zeta_{1}, \cdots, \zeta_{n-1}\right\}$ such that $\max \left\{\left\|\zeta_{i}-\xi_{i}\right\|,\left\|\zeta_{i}^{-1}-\xi_{i}^{-1}\right\|\right\}<\epsilon$, for any $i=0,1, \cdots, n-1$, and $\operatorname{det}\left(\mathcal{C}_{\mathcal{A}}\right)=\operatorname{det}\left(\mathcal{C}_{\mathcal{B}}\right)$ and the eigenvalues of $\mathcal{C}_{\mathcal{B}}$ are all real, and have same modulus.

To prove Theorem B, we need another lemma about uniformly contracting family. Let $\mathcal{A}=\left\{\xi_{i}: 0 \leq i \leq k-1\right\} \subset G L(n)$ be a periodic family. We say the sequence $\mathcal{A}$ is uniformly contracting family if there is a constant $\delta>0$ such that for any $\delta$-perturbation of $\mathcal{A}$ are sink,.i.e, for any $\mathcal{B}=\left\{\zeta_{i}: 0 \leq i \leq k-1\right\}$ with $\left\|\zeta_{i}-\xi_{i}\right\|<\delta$, all eigenvalue of $\mathcal{C}_{\mathcal{B}}$ have moduli less than 1. Similarly, we can define the uniformly expanding periodic family. The following theorem is well known.

Theorem $2.4([7])$. For any $\delta>0$ and $K>0$, there are constants $C>0,0<\lambda<1$ and positive integer $m$ such that if $\mathcal{A}=\left\{A_{0}, A_{1}, \ldots, A_{n-1}\right\}$ is a uniformly contracting periodic family which satisfies

$$
\max _{i=0,1, \ldots, n-1}\left\{\left\|A_{i}\right\|,\left\|A_{i}^{-1}\right\|\right\}<K
$$

for $n>m$, then 


$$
\prod_{j=0}^{k-1}\left\|\prod_{i=0}^{m-1} A_{i+m j}\right\| \leq C \lambda^{k}
$$

where $k=[n / m]$.

\section{Proof of Theorem B}

Let $M$ be as before, and let $f \in \operatorname{Diff}(M)$. In this section, we will use the notation of pre-sink (resp. pre-source). A periodic point $p$ is called a pre-sink (resp. pre-source) if $D f^{\pi(p)}(p)$ has an multiplicity one eigenvalue equal to +1 or -1 and the other eigenvalues has norm less than 1 (resp. bigger than 1).

Remark 3.1 ([1, Theorem 2.2.23 and 2.2.26]). Let $f \in \operatorname{Diff}(M)$.

- Let $\mathcal{I}$ be an small arc. Then $f: \mathcal{I} \rightarrow \mathcal{I}$ is not expansive.

- Let $\mathcal{C}$ be a small circle. Then $f: \mathcal{C} \rightarrow \mathcal{C}$ is not expansive.

Recall that if $\Lambda$ is $C^{1}$-stably expansive then there are a $C^{1}$-neighborhood $\mathcal{U}(f)$ and a compact neighborhood $U$ of $\Lambda$ such that for any $g \in \mathcal{U}(f), \Lambda_{g}(U)=\bigcap_{n \in \mathbb{Z}} g^{n}(U)$ is expansive for $g$.

Lemma 3.2. Let $\Lambda$ be a closed set of $f \in \operatorname{Diff}(M)$, and let $\mathcal{U}(f)$ and $U$ be as above. If $\Lambda$ is $C^{1}$-stably expansive, then for any $g \in \mathcal{U}(f), g$ has neither pre-sink nor pre-source with the orbit staying in $U$.

Proof. Suppose that $f$ is $C^{1}$-stably expansive on $\Lambda$. Then there are a $C^{1}$-neighborhood $\mathcal{U}(f)$ of $f$ and a compact neighborhood $U$ of $\Lambda$ such that for any $g \in \mathcal{U}(f)$, $g$ is expansive on $\Lambda_{g}(U)=\bigcap_{n \in \mathbb{Z}} g^{n}(U)$. Assume that there is $g \in \mathcal{U}(f)$ such that $g$ has a pre-sink $p$ with $\mathcal{O}(p) \subset U$. For simplicity, we may assume $p$ is fixed point of $g$ (other case is similar).

By making use of the Franks' Lemma, we linearize $g$ at $p$ with respect to the exponential coordinates $\exp _{p}$, i.e, choose $\epsilon_{1}>0$ and $\alpha>0$ with $B_{\alpha}(p) \subset U$ and there exists $g_{1} C^{1}-\epsilon_{1}$ nearby $g$ such that

$$
g_{1}(x)= \begin{cases}\exp _{p} \circ D_{p} g(p) \circ \exp _{p}^{-1}(x) & \text { if } x \in B_{\alpha}(p), \\ g(x) & \text { if } x \notin B_{4 \alpha}(p) .\end{cases}
$$

Then $g_{1}(p)=g(p)=p$.

Since $p$ is pre-sink of $g, D_{p} g$ has a multiplicity one eigenvalue such that $|\lambda|=1$ and other eigenvalues of $D_{p} g$ are with modulus less than 1 . Denote by $E_{p}^{c}$ the eigenspace corresponding to $\lambda$, and $E_{p}^{s}$ the eigenspace corresponding to the eigenvalues with modulus less than 1 . Thus $T_{p} M=E_{p}^{c} \oplus E_{p}^{s}$. Then we get two cases: $\lambda$ is real or complex. 
Case 1: $\lambda$ is real. Then $\operatorname{dim} E_{p}^{c}=1$. For simplicity, we suppose that $\lambda=1$. There is a small arc $\mathcal{I}_{p} \subset B_{\alpha}(p) \cap \exp _{p}\left(E_{p}^{c}(\alpha)\right)$ center at $p$ such that $\left.g_{1}\right|_{\mathcal{I}_{p}}=i d$, where $i d$ is identity map. Here $E_{p}^{c}(\alpha)$ is the $\alpha$-ball in $E_{p}^{c}$ center at the origin $O_{p}$. Clearly, $\mathcal{I}_{p} \subset \Lambda_{g_{1}}(U)$.

Note that for a set $A \subset M$, if $M$ is expansive then $A$ have to expansive. By the definition of the $C^{1}$-stably expansivity, $\left.g_{1}\right|_{\Lambda_{g_{1}}(U)}$ is expansive. Moreover, by Remark $3.1,\left.g_{1}\right|_{\mathcal{I}_{p}}$ is not expansive. This is a contradiction. Therefore, if $\Lambda$ is $C^{1}$-stably expansive of $f$ then it does not have pre-sink.

Case 2: $\lambda$ is complex. Then $\operatorname{dim} E_{p}^{c}=2$. Since the eigenvalue $\lambda$ is complex, there is a small circle $\mathcal{C}_{p} \subset B_{\alpha}(p) \cap \exp _{p}\left(E_{p}^{c}(\alpha)\right)$ center at $p$ such that $\left.g_{1}\right|_{\mathcal{C}_{p}}$ is conjugate to an irrational rotation map. Here $E_{p}^{c}(\alpha)$ is the $\alpha$-ball in $E_{p}^{c}$ center at the origin $O_{p}$. Clearly, $\mathcal{C}_{p} \subset \Lambda_{g_{1}}(U)$. Thus by the notion of $C^{1}$-stably expansivity, $\left.g_{1}\right|_{\mathcal{C}_{p}}$ has to be expansive. Again by Remark 3.1, the rotation map $g_{1}: \mathcal{C}_{p} \rightarrow \mathcal{C}_{p}$ is not expansive. This is a contradiction.

Therefore, if $\Lambda$ is $C^{1}$-stably expansive of $f$ then it does not have pre-sink. Similarly, $f$ does not have pre-source.

The following lemma is well known result. In fact, we make using the $C^{1}$-closing lemma and property of transitive set. Hereafter, we consider transitive sets is nontrival, that is, the set is not one orbit.

Lemma $3.3([8])$. Let $\Lambda$ be a transitive set. There exist a sequence $\left\{g_{n}\right\}_{n \in N}$ of diffeomorphism and a periodic orbit $P_{n}$ of $g_{n}$ with period $\pi\left(P_{n}\right) \rightarrow \infty$ as $n \rightarrow \infty$ such that $g_{n} \rightarrow f$ in the $C^{1}$-topology and $\lim _{H} P_{n}=\Lambda$, where $\lim _{H}$ is the Hausdorff limit and $\pi\left(P_{n}\right)$ is the period of $P_{n}$.

From Lemma 3.3 , we can choose $p_{n} \in P_{n}$ such that we get a periodic family $\mathcal{A}_{n}=\left\{D_{p_{n}} f, D_{f\left(p_{n}\right)} f, \ldots, D_{f^{\pi\left(p_{n}\right)-1}\left(p_{n}\right)} f\right\}$.

Lemma $3.4([4])$. Let $\Lambda, P_{n}$ be as in Lemma 3.3, and $\mathcal{A}_{n}$ be given as above. Then for any $\epsilon>0$ there exists an integer $n_{0}(\epsilon)>0$ such that for any $n>n_{0}(\epsilon), \mathcal{A}_{n}$ is neither $\epsilon$-uniformly contracting nor $\epsilon$-uniformly expanding.

Let $\mathcal{U}_{0}(f)$ be given by Lemma 3.2, and let $g \in \mathcal{U}_{0}(f)$. We consider the periodic family of linear maps $\mathcal{A}=\left\{D_{p} g\right.$ : for any $\left.p \in P(g) \cap \Lambda_{g}(U)\right\}$. Let $\mathcal{B}=\left\{\xi_{p}\right.$ : for any $\left.p \in P(g) \cap \Lambda_{g}(U)\right\}$ be a family of periodic sequence of linear maps closed to $\mathcal{A}$, and for any $p \in P(g) \cap \Lambda_{g}(U)$, consider the linear map

$$
\mathcal{C}_{\mathcal{B}}=\xi_{g^{\pi(p)-1}(p)} \circ \cdots \circ \xi_{p},
$$


and denote by $\lambda_{s}\left(\mathcal{C}_{\mathcal{B}}\right), \lambda_{u}\left(\mathcal{C}_{\mathcal{B}}\right)$ its eigenvalues. Here $\xi_{g^{i}(p)}$ is a linear map nearby $D_{g^{i}(p)} g$ for $0 \leq i \leq \pi(p)-1$ and $\left|\lambda_{s}\left(\mathcal{C}_{\mathcal{B}}\right)\right| \leq\left|\lambda_{u}\left(\mathcal{C}_{\mathcal{B}}\right)\right|$.

Lemma $3.5([4])$. Let $\Lambda, P_{n}$ be as in Lemma 3.3. Then for any $\epsilon>0$ there are $n(\epsilon), l(\epsilon)>0$ such that for any $n>n(\epsilon)$ if $P_{n}$ does not admits a $l(\epsilon)$ dominated splitting, then choose $g C^{1}$-nearby $f$ and preserving the orbit of $P_{n}$ such that $P_{n}$ is pre-sink or pre-source respecting $g$.

From Lemma 3.2 and Lemma 3.5, we can get the following Proposition 3.6.

Proposition 3.6. Let $\Lambda$ be a transitive set. Then if $\Lambda$ is $C^{1}$-stably expansive, then we can choose $N, l>0$ such that for any $n>N, P_{n}$ admits a l-dominated splitting.

Proof. Let $\Lambda$ be a transitive set. Suppose that $\Lambda$ is $C^{1}$-stably expansive. Then by Franks' Lemma, and by the notion of the $C^{1}$-stably expansivity, there are a $C^{1}$ neighborhood $\mathcal{U}(f)$ of $f$ and a compact neighborhood $U$ of $\Lambda$ such that for any $g \in \mathcal{U}_{0}(f) \subset \mathcal{U}(f),\left.g\right|_{\Lambda_{g}(U)}$ is expansive. By Lemma 3.2, $g$ has neither pre-sink nor pre-source. And, by Lemma 3.5, $P_{n}$ is neither pre-sink nor pre-source respecting $g$. Therefore, by Lemma 3.5, $P_{n}$ admits a $l$-dominated splitting.

By Proposition 3.6 and the following proposition, we directly obtain Theorem B.

Proposition $3.7([2])$. Let $g_{n}$ convergent to $f$ and if $\Lambda_{g_{n}}$ be a closed $g_{n}$-invariant set of $g_{n}$ and $\lim \Lambda_{g_{n}}=\Lambda$. Then if $\Lambda_{g_{n}}$ admits a l-dominated splitting respecting $g_{n}$, then $\Lambda$ admits a l-dominated splitting respecting $f$.

End of proof of Theorem $B$. Let $\Lambda$ be a transitive set of $f \in \operatorname{Diff}(M)$. Then by Lemma 3.3, there exists a sequence $\left\{g_{n}\right\}_{n \in \mathbb{Z}}$ of diffeomorphism and a periodic orbit $P_{n}$ of $g_{n}$ such that $g_{n} \rightarrow f$ in the $C^{1}$-topology and $P_{n} \rightarrow \Lambda$ in the Hausdorff limit. By Proposition 3.6, $P_{n}$ admits a $l$-dominated splitting. Thus by Proposition 3.7, $\Lambda$ admits a $l$-dominated splitting.

\section{REFERENCES}

1. N. Aoki \& K. Hiraide: Topological Theory of Dynamical Systems. Recent Advances. North-Holland Math. Library 52 North-Holland, Amsterdam 1994.

2. C. Bonatti, L.J.Díaz \& E. Pujals: A $C^{1}$-generic dichotomy for diffeomorphisms: weak forms of hyperbolicity or infinitely many sinks or sources. Annals of Math. 158 (2003), 187-222.

3. C. Bonatti, N. Gourmelon \& T. Vivier: Perturbations of the derivative along periodic orbits. Ergodi. Th. \& Dynm. Syst. 26 (2006), 1307-1337. 
4. K. Lee, G. Lu \& X. Wen: $C^{1}$-stably weak shadowing property of chain transitive sets. preprint.

5. K. Lee \& M. Lee: Hyperbolicity of $C^{1}$-stably expansive homoclinic classes. Disc. \& Contin. Dynam. Syst. 27 (2010), 1133-1145.

6. R. Mãné: Expansive diffeomorphisms. Lecture Notes in Math. 468, Springer-Verlag (1975), 162-174.

7. _ : An ergodic closing lemma. Ann. Math. 116 (1982), 503-540.

8. D. Yang: Stably weakly shadowing transiteve sets and dominated splitting. Proc. Amer. Math. Soc. 139 (2011), 2747-2751.

Department of Mathematics, Mokwon University, Daejeon, 302-729, Korea

Email address: Imsds@mokwon.ac.kr 